\title{
Interactive Visualization of Breast Shape for Breast Surgery
}

\author{
Urmila SAMPATHKUMAR ${ }^{1}$, Audrey CHEONG $^{2}$, Gregory P. REECE ${ }^{3}$, Mary Catherine BORDES ${ }^{3}$, \\ Summer E. HANSON ${ }^{3}$, Mia K. MARKEY ${ }^{4,5}$, Fatima A. MERCHANT ${ }^{* 1,2,4,6}$ \\ ${ }^{1}$ Department of Computer Science, University of Houston, TX, USA; \\ ${ }^{2}$ Department of Electrical and Computer Engineering, University of Houston, TX, USA; \\ ${ }^{3}$ Department of Plastic Surgery, The University of Texas MD Anderson Cancer Center, TX, USA; \\ ${ }^{4}$ Department of Biomedical Engineering, The University of Texas at Austin, Austin, TX, USA; \\ ${ }^{5}$ Department of Imaging Physics, The University of Texas MD Anderson Cancer Center, TX, USA; \\ ${ }^{6}$ Department of Engineering Technology, University of Houston, TX, USA
}

\section{https://doi.org/10.15221/19.034}

\begin{abstract}
While advancements in plastic surgery techniques and technology have facilitated improved aesthetic outcomes in both cosmetic and reconstructive breast surgery, communicating potential outcomes to the patient prior to the surgery, and understanding their aesthetic preferences for breast shape and size still remains a challenge. Unrealistic expectations related breast aesthetics can lead to patients' dissatisfaction with surgical outcomes. Currently, plastic surgeons rely on drawings or images of former patients to explain surgical procedures and their possible outcomes. The ability to visualize the expected post-surgery breast would, to a large extent, mitigate this challenge and aid both surgical planning, and inform decision making. In this work we propose a software tool that will enable visualization of three-dimensional (3D) images of patient's breasts and allow real time simulation of morphological changes on the breast.

3D surface images of the patients' torso were obtained using stereophotogrammetry during their clinical visits. The front torso area between the sternal notch and the umbilicus was captured. The breast region of interest was extracted semi-automatically from the 3D surface mesh of the torso and a computational model using Fourier based spherical harmonics (SPHARM) was created. SPHARM coefficients obtained from modeling were previously observed to have direct correlation with breast size and shape measurements, such as height, width, projection and ptosis. Simulation of breast shape changes was achieved by modifying these coefficients. We designed an application for visualization of the simulated breast on the 3D surface image of the torso with real time simulation of breast size changes by modifying the model parameters. The work in this study addresses design and development of the visualization software. Future work will focus on evaluating the visualization framework to assess surgeon and patient acceptance on usability, and feasibility for clinical translation.
\end{abstract}

Keywords: Breast surgery, Breast shape visualization, 3D torso image, Breast shape simulation

\section{Introduction}

Breast shape may greatly affect body image in some women. Studies have shown that some women are dissatisfied with their breast size and shape [1]. Cosmetic and reconstructive breast surgeries aim to revise or restore breast aesthetics. For these surgeries, breast shape aesthetic is thus a critical outcome. The ability to visualize a patient's expectations related to changes in breast shape and size that they desire could help surgeons understand individual patient preferences for breast aesthetics. Moreover, while advancements in plastic surgery techniques and technology have facilitated improved aesthetic outcomes in breast surgery, they have also resulted in more medically viable surgical options for patients. Computational tools for simulating and presenting possible surgical outcomes for different surgical procedures would facilitate surgeon-patient communication related to breast aesthetics. Appropriately eliciting patient preferences related to breast aesthetics and communicating possible surgical outcomes for breast shape and size are two key challenges encountered during clinical consultations for breast surgeries.

Breast reconstruction surgery is typically performed on cancer patients who have undergone a total mastectomy. In reconstructive surgery, a patient might have to go through intermediate procedures such as the placement of tissue expanders. It is important to inform and educate patients on how their breasts will appear/change during the course of reconstruction. Currently, surgeons rely on hand-drawn illustrations or two-dimensional (2D) photos to explain surgical procedures and possible outcomes to patients. 2D images are restrictive in dimensionality and fail to portray changes to overall breast size and shape in a single image. In contrast, a single 3D image provides the advantage to view the breast from multiple angles. Donfrancesco et al., investigated the utility of 3D simulations during clinical

*fmerchant@central.uh.edu; +1-832-722-7286 
consultation prior to breast augmentation surgery [2]. Out of 150 participants, $55 \%$ reported that 3D simulations played a very important role throughout their breast augmentation experience, $34 \%$ reported 3D simulations were important, and $11 \%$ indicated that 3D simulations were not very important. Overall $83 \%$ of the patients indicated that 3D simulation influenced their decision to undergo surgery. $A$ $3 \mathrm{D}$ visualization tool could prove useful in effectively communicating to the patient, possible changes to breast appearance that could occur during various stages of breast surgery.

In this work, we designed an application for visualization of 3D surface images of the torso in conjunction with simulations to enable real time display of changes in breast size. Fourier spherical harmonics (SPHARM) functions were used to model the 3D breast shape and the model parameters were modulated to simulate changes in breast size.

While a number of studies have used 3D imaging to simulate and study results of surgical procedures [3-6], few have developed user-friendly visualization and simulation tools that can be used in a clinical setting for pre-surgery consultation. Oren et al., used 3D imaging and visualization tools to assess the outcome of unilateral tissue expander-implant breast reconstruction surgery [6]. However, they did not provide a visualization interface or 3D simulation of breast shape changes for pre-surgical consultation.

Roose et al., have developed a biomechanical breast augmentation simulation methodology for use in surgical planning [7]. Ciechomski et al., developed a web-based consultation tool [8] that utilizes several $2 \mathrm{D}$ images of the patient's torso taken at 3 different angles with manually annotated fiducial points to reconstruct a 3D model of the breasts. The tool then uses biomechanical simulations to visualize possible breast augmentation results. The tool allows the surgeons to choose implants from a catalogue and also control the diameter and placements of the implants in the simulation. Although the tool appears promising it can only simulate breast augmentation procedures.

Other commercially available software, such as Vectra 3D [9] and ILLUSIO [10] also offer functionality for modulating breast shape. Vectra 3D enables visualization, measurement, and simulations of breast surgery results using computational models. ILLUSIO software uses augmented reality to overlay virtual breasts on the patient's body, allowing the patient to view on a virtual mirror their post-surgical reflection. It allows the surgeon to manipulate the model as the patient is viewing the image and also allows the patient to turn from side-to-side to visualize the results from different angles. However, publications discussing the underlying methodology for breast shape modulation, or providing a comprehensive assessment in terms of performance, usability and clinical feasibility are lacking.

\section{Materials and Methods}

\subsection{Participants}

We have previously collected 3D surface torso images from healthy commissioned volunteers and a cohort of 505 patients who underwent mastectomy followed by breast reconstruction at The University of Texas MD Anderson Cancer Center from 2011-2014 (IRB approved protocol). 3D surface torso images were taken with a 3dMDTorso system (3dMD Inc., Atlanta, GA, USA) over various time points from before reconstruction to $18+$ months post-reconstruction. For this study, we selected a group of patients for whom baseline images of native breasts prior to reconstruction were available, and images from commissioned volunteers. Each 3D image is represented in the torso as triangular mesh with an associated $2 \mathrm{D}$ texture. Only the frontal portion of the torso is captured during imaging and the resultant $3 \mathrm{D}$ mesh is not closed.

\subsection{Breast Shape Modeling}

In our previous work [11], we used Fourier spherical harmonics (SPHARM) based computational method to model breast shape from 3D surface images of 161 breasts from 87 patients. Spherical harmonics are a series of orthogonal functions defined on the surface of a unit sphere. The approach utilizes 3D surface images of the female torso, extracts the breast regions, and represents breast shape in terms of spherical harmonics. The SPHARM method converts a 3D object of spherical topology into three sets of SPHARM coefficients that describe its overall shape in terms of three sets of spherical harmonics basis functions (one set for each dimension). Analysis of SPHARM coefficients obtained from SPHARM modeling of the breasts has shown direct correlation between the SPHARM coefficients and the breast dimensions such as height, width, projection, and ptosis (breast sagging) [11]. Modulating the SPHARM coefficients enables us to simulate changes to breast dimensions. 
The region of interest $(\mathrm{ROI})$ consisting of a single breast was cropped from the $3 \mathrm{D}$ torso surface using a semi-automated process. For ROI cropping, the fiducial points - Midline point (ML), Transition point (TP), and Lateral point (LP) were manually annotated. The TP is the point along the meridian line from the mid-clavicle to the nipple, where the upper chest wall transitions to the breast. The medial point is where the inframammary fold (the natural lower boundary of the breast), ends medially. The ML is located midway between the medial points of the left and right breasts. The LP is the point where the inframammary fold ends laterally. The Inferior Breast-Chest contour (IBC), which is the lowest visible boundary of the breast, was detected using a contour detection algorithm [12]. The breast was cropped using the annotated fiducial points and the detected IBC. Since SPHARM functions can be applied only on closed surfaces, the cropped breast surface was closed using a computationally generated back plane. The breast is represented as triangular mesh with vertices $x, y, z$ in Cartesian co-ordinate system. To apply SPHARM expansion, the vertices have to be converted to a parametric domain. A custom parameterization algorithm [11] was developed to obtain spherical topology and standardize breast orientation. The parameterization maps every vertex on the breast surface onto the surface a unit sphere $\left(\theta=\left[-\frac{\pi}{2}, \frac{\pi}{2}\right]\right.$ and $\left.\emptyset=[-\pi, \pi]\right)$ with the most projecting point (MPP) set at $\theta=\pi / 2$. The vertex $x, y, z$ was mapped to $x(\theta, \varnothing), y(\theta, \varnothing), z(\theta, \varnothing)$ respectively using radial function $r(\theta, \varnothing)$ described in $[11,13]$.

SPHARM basis expansion was then applied to the parameterized breast surface to estimate the SPHARM coefficients. Fourier spherical harmonics function of degree $l$ and order $m$ is given by equation (1)

$$
Y_{l}^{m}(\theta, \varnothing)=\sqrt{\frac{(2 l+1)(l-m) !}{4 \pi(l+m) !}} P_{l}^{m}(\cos \theta) e^{i m \varnothing}
$$

where $P_{l}^{m}(\cos \theta)$ is the associated Legendre polynomial described in [14]. The parameterized vertex $x(\theta, \varnothing), y(\theta, \varnothing), z(\theta, \varnothing)$ was computed using

$$
v(\theta, \varnothing)=\sum_{l=0}^{\infty} \sum_{m=-l}^{l} c_{l}^{m} Y_{l}^{m}(\theta, \varnothing)
$$

Where $v(\theta, \varnothing)=(x(\theta, \varnothing), y(\theta, \varnothing), z(\theta, \varnothing))^{T}$ and $c_{l}^{m}=\left(c_{l x}^{m}, c_{l y}^{m}, c_{l z}^{m}\right)^{T}$ are the estimated SPHARM coefficients.

SPHARM-MAT $[15,16]$ implementation was used for parametrization and expansion. The expansion results in 1320 (440X3) SPHARM coefficients that uniquely describe breast shape.
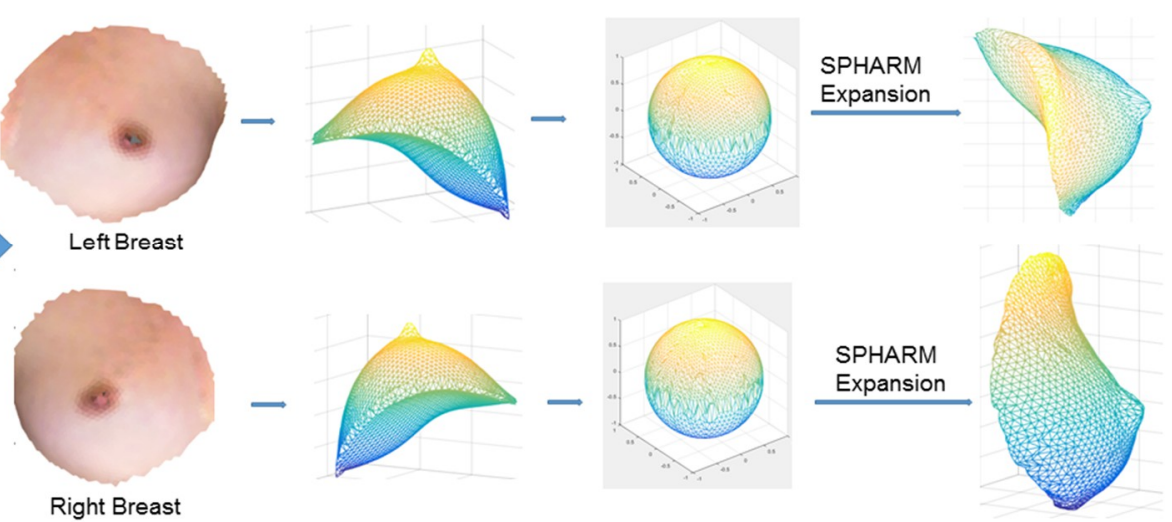

Fig. 1. SPHARM Modeling steps. (a) Fiducial points are manually marked. (b) Output from the breast crop algorithm. (c) Genus zero object formed by closing the breast surface using a computationally generated back plane. (d) Parameterization. Breast vertices mapped onto the surface of a unit sphere. (e) Breast mesh reconstructed from SPHARM model, which is obtained by applying SPHARM expansion function on the parameterized surface.

The SPHARM coefficients obtained from the modeling are the shape descriptors of the modeled breast surface. SPHARM coefficients were previously found to be linearly correlated with the breast dimensions such as height, width, and projection, and ptosis [11]. 


\subsection{Visualization Framework}

A graphical user interface (GUI) was built in Matlab (The MathWorks, Inc., Natick, MA) to enable visualization of breast shape modulation based on SPHARM modeling. The application enables real time changes to breast size by changing related coefficients in the SPHARM model. The application takes a breast SPHARM model and user provided breast measurements as inputs to generate the modified breast vertices. The GUI (Figure 2) visualizes the breast image generated from the given SPHARM model. The baseline breast measurements are automatically computed and displayed. Height was measured as the distance between the two extreme breast vertices along the Z-axis, width measured as the distance between the two extreme breast vertices along the $Y$-axis and projection was measured as the distance between the two extreme breast vertices along the $\mathrm{X}$-axis. The ptosis value shown in the GUI is the relative change applied to the computed SPHARM coefficient. The correspondence of this value to standardized ptosis grades is not included in this study and will be implemented in future work. During interactive modulation, the software only enables visualization of changes in the sagging of the breast relative to the original breast shape.

The slider elements of the GUI enable the user to dynamically change the breast dimensions. As the user changes the breast measurements, corresponding SPHARM coefficients are changed and the breast vertices are recomputed with the new coefficients. The change in the breast size is displayed in near real time with minimal to no time lag for computation and rendering. This makes SPHARM based breast shape modulation a potential candidate for simulation in clinical settings.

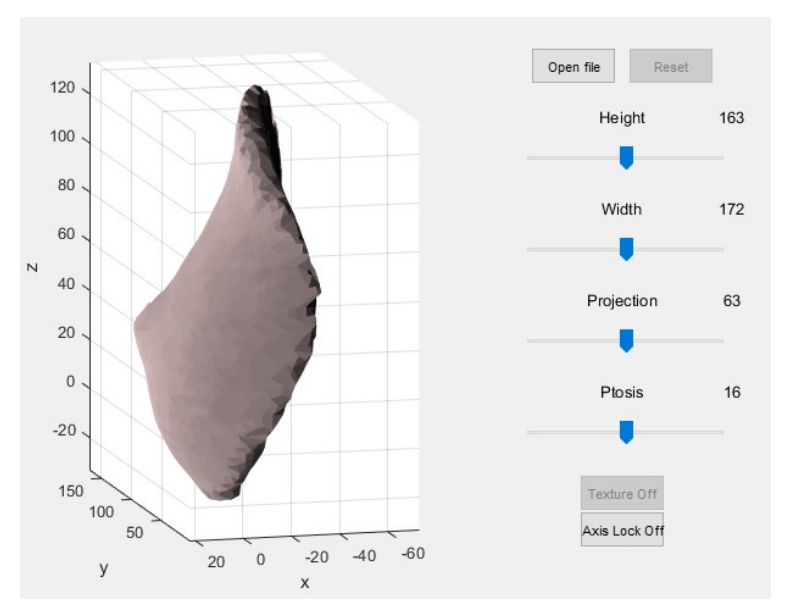

Fig. 2. Graphical User Interface of the Matlab application to change breast dimensions Breast size can be manipulated using the sliders.

We established the feasibility of SPHARM modeling to change the breast dimensions and simulate changes in breast shape as discussed above. SPHARM modeling can be potentially applied to visualize possible post-operative outcomes and for the elicitation of patient preferences with regards to the breast size.

Next, we also developed a C++ application using the Visualization Toolkit (VTK) [17] for 3D mesh rendering and manipulation, and Qt libraries [18] for GUI development. In contrast to the Matlab GUI that enables visualization of the cropped breast, the $\mathrm{C}++$ visualization framework enables display and manipulation of the 3D image of the torso. The GUI consist of 3 parts a) Menu bar, b) Rendering area c) Interaction Tools. The menu bar located in the top left corner of the GUI provides menu buttons to browse and select an input 3D image file. The selected patient image is displayed in rendering area (as seen in the left panel of the GUI in Figure 4). The rendering is interactive, wherein, the user can pan, zoom, translate and rotate the 3D image. Interactive radio buttons allow the user to choose a breast (left or right), while slider components allow the user to dynamically change breast dimensions. 


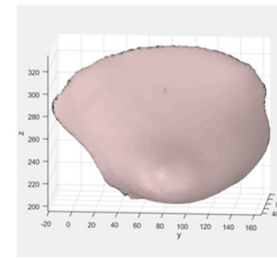

Width $=162 \mathrm{~mm}$

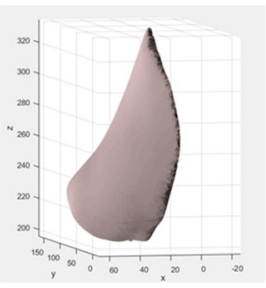

Baseline ptosis

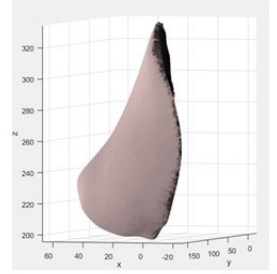

$$
\text { Projection }=63 \mathrm{~mm}
$$

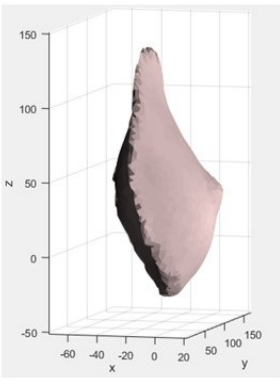

Height $=163 \mathrm{~mm}$

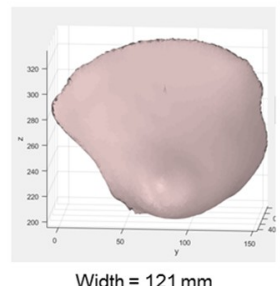

Width $=121 \mathrm{~mm}$

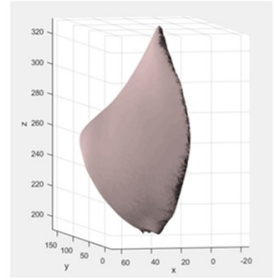

No Ptosis

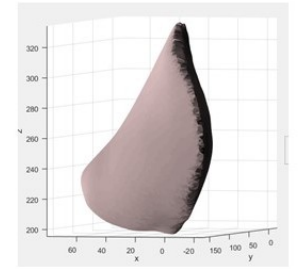

Projection $=111 \mathrm{~mm}$

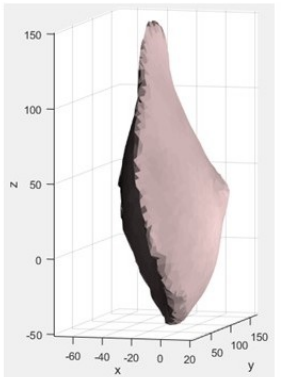

Height $=213 \mathrm{~mm}$

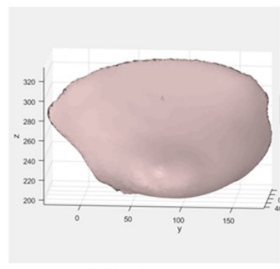

Width $=212 \mathrm{~mm}$

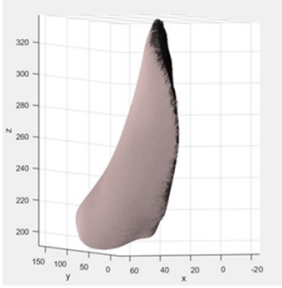

Grade 1 Ptosis

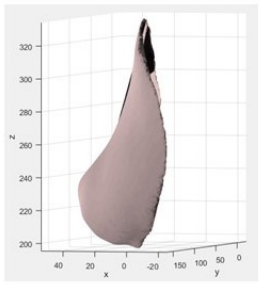

Projection $=15 \mathrm{~mm}$

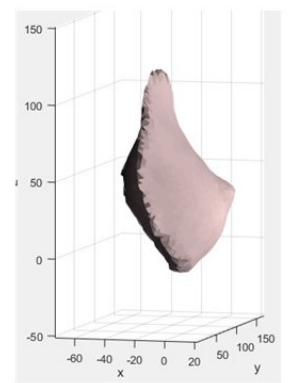

Height $=113 \mathrm{~mm}$

Fig. 3. Images showing width, ptosis, projection, and height change simulations using the Matlab prototype.
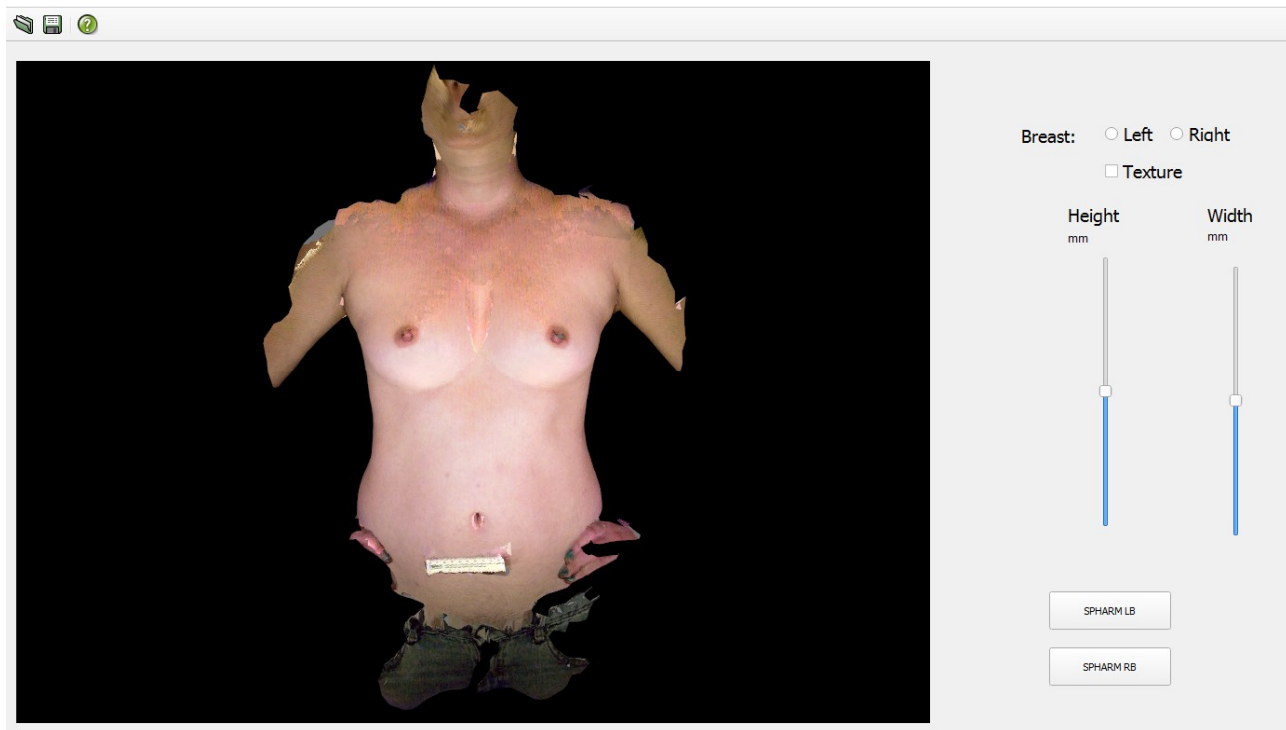

Fig. 4. Graphical User Interface of the C++ visualization framework. 
The visualization framework is comprised of the following three components: 1) Qt for developing the GUI, 2) Visualization Toolkit (VTK) for rendering of the 3D image and 3) Matlab runtime compiled library function for modification of SPHARM coefficients.

Qt is an open source cross platform application development framework written in C++ for desktop, embedded and mobile computing, that is commonly used for creating GUls. VTK is an open-source OpenGL based C++ library used to develop 3D computer graphics, image processing and scientific visualization applications. Matlab runtime is used to execute compiled Matlab applications without installing commercial Matlab software.

Figure 5 presents the flowchart for development of the visualization framework. The visualization tool utilizes two input files, the 3D torso image and the modeled SPHARM coefficients for one of the breasts (left or right). The user interacts with the GUI created using Qt. When a user requests to view a 3D image (Extensible 3D (X3D) format images permissible), the requested file is read and passed to VTK for rendering. X3D is an open standard file format to represent a 3D scene. The X3D files consists of vertices, edges and polygon information to render the surface mesh. It also specifies texture coordinates whose values can be retrieved from the complimentary texture image. The 3D image is sent to the VTK that renders the image in a VTK render widget inside the Qt GUI. SPHARM models are precomputed in Matlab using the method described earlier. When the user specifies the name of the precomputed SPHARM model files, the Matlab runtime environment invokes a function that reads the SPHARM input file and returns the SPHARM model. To visualize the breast model on the torso, re-rendering of the torso surface mesh is performed after replacing the breast ROI mesh vertices by the SPHARM breast model vertices. As the user makes changes to breast dimensions, the requested change is sent back to the Matlab functions that change the corresponding SPHARM coefficients and return a modified SPHARM model to be re-rendered and displayed.

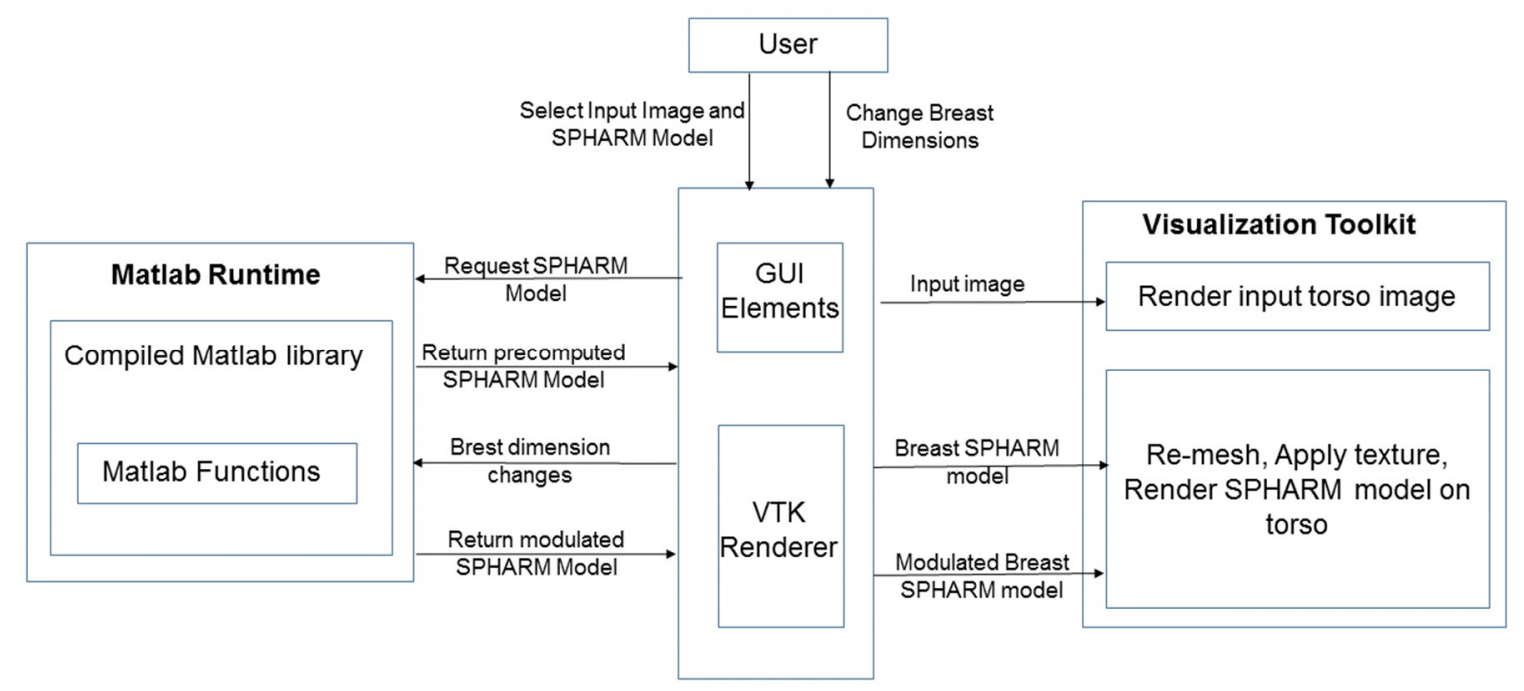

Fig. 5. Flowchart of visualization framework.

\section{Results}

Figure 6(a) shows a SPHARM model of the patient's native breast at baseline visualized on the torso. Figure $6(\mathrm{~b})$ is the frontal view of the torso showing a simulated right breast model following changes made to the height, width and projection of the original baseline model in Figure 6(a) to increase its size. Similarly Figure 6(c) presents a simulation of the right breast model following changes made to the height, width and projection of the original baseline model in Figure 6(a) to reduce its size. Additionally, changes were applied to the left breast of the same patient and results of simulated left breasts are shown in Figures 7(b) and (c). Figure 8 shows representative images of a commissioned volunteer with modifications visualized in the lateral view. Mesh manipulation is a challenging task and is susceptible to failure in some cases. An example image illustrating failure of the software to generate and render a smooth mesh surface is shown in Figure 9. This occurs when large changes to breast dimensions are implemented, resulting in increased displacement of the breast vertices and consequently generation of enlarged face triangles in the surface mesh. The displacement of points may lead to few surface triangles projecting out of the mesh with consequent artifacts in texture mapping. This problem can be mitigated by re-computing the surface mesh by interpolating additional vertices to break down larger face triangles into several small ones. 


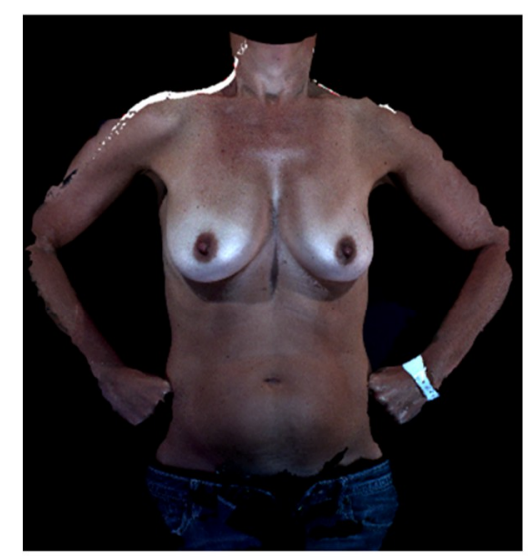

(a) Baseline

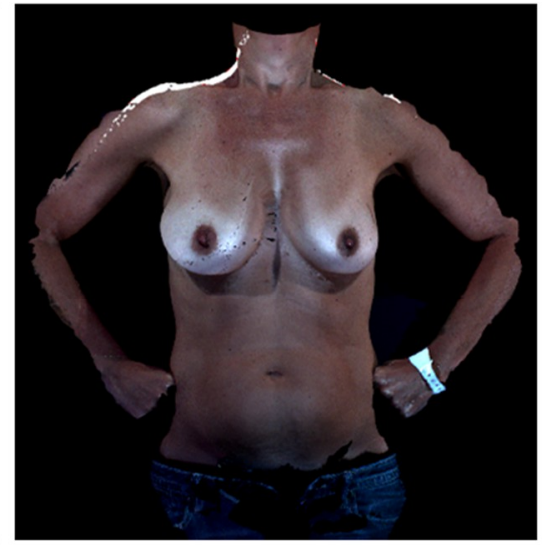

(b) Right breast increased

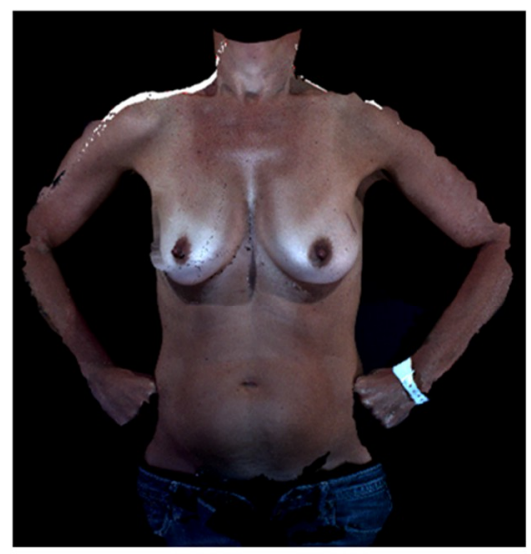

(c) Right breast reduced

Fig. 6 Visualization of breast size changes to the right breast using the developed tool. (a) Baseline image.

(b) Right breast increased in size after changes to height, width, depth and ptosis. (c) Right breast reduced in size after changes to height, width, depth and ptosis

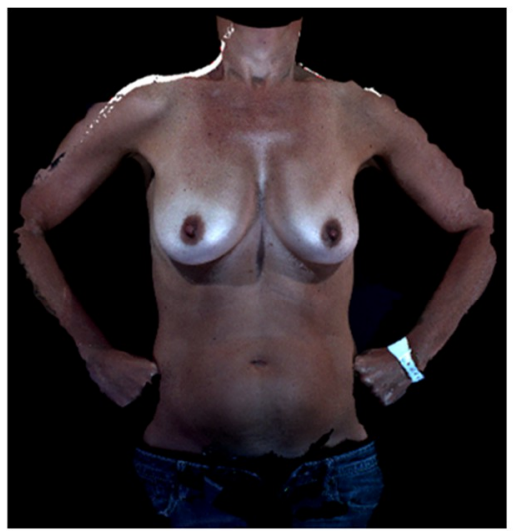

(a) Baseline

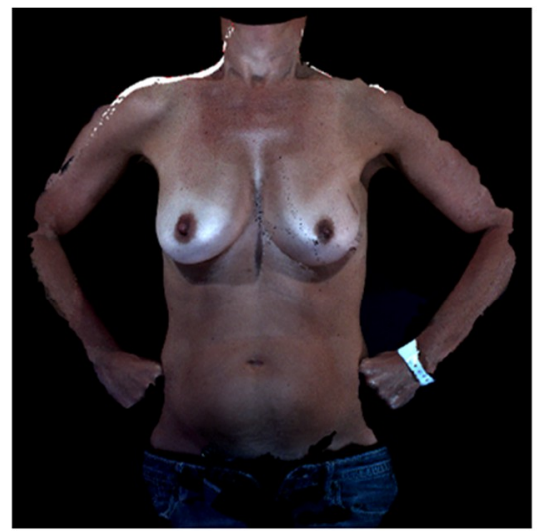

(b) Left breast increased

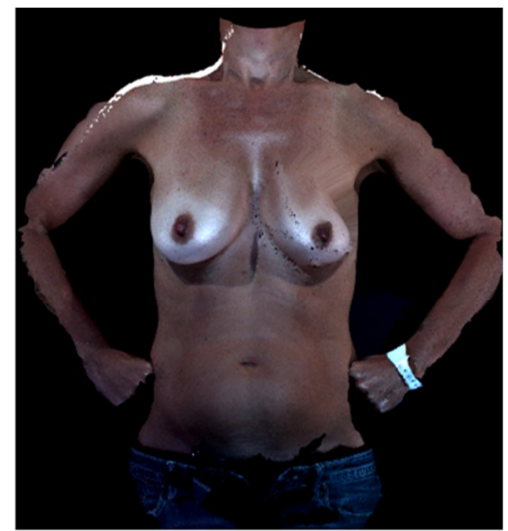

(c) Left breast reduced

Fig. 7. Visualization of breast size changes to the left breast using the developed tool. (a) Baseline image. (b) Left breast increased in size after changes to height, width, depth and ptosis. (c) Left breast reduced in size after changes to height, width, depth and ptosis

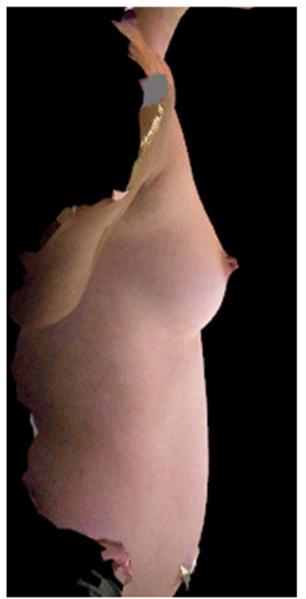

(a) Baseline

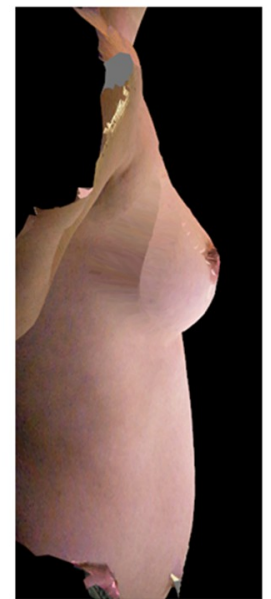

(b) Right breast increased

Fig. 8. Lateral view of changes in breast size using the developed visualization tool.

(a) Baseline image and (b) Image showing changes made to the right breast to increase its size 


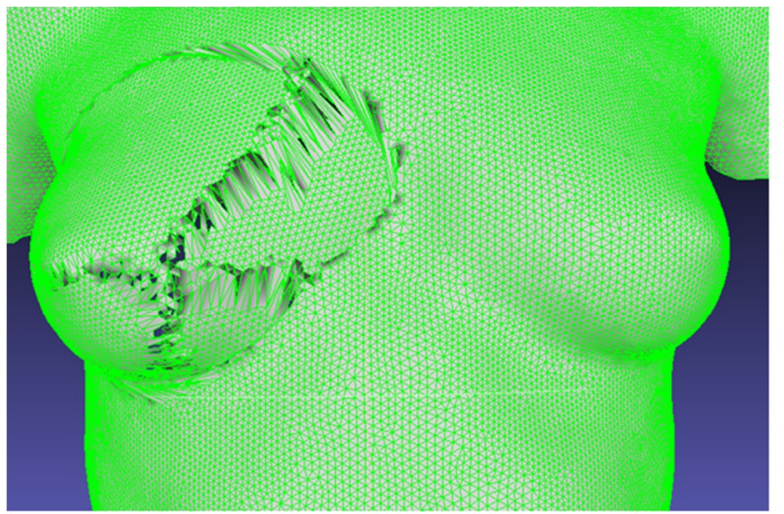

(a) Surface mesh re-rendering may result in enlarged face triangles

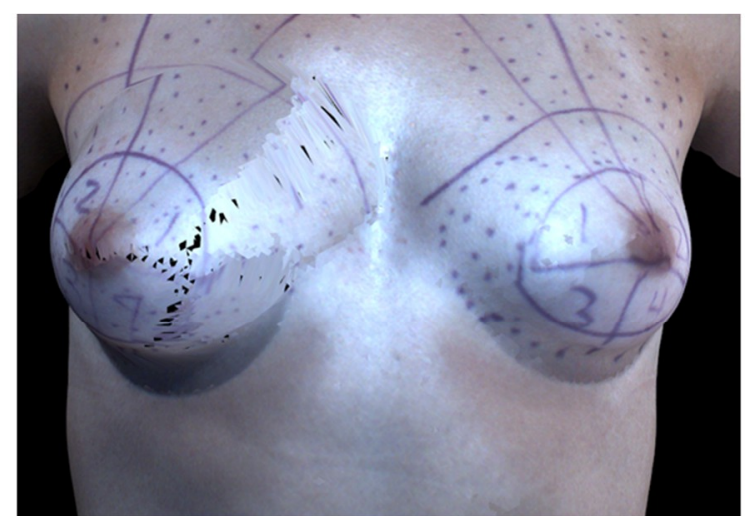

(b) Artifacts introduced in texture mapping due to enlarged triangles in re-rendered surface mesh

Fig. 9. Representative images illustrating complexity of surface mesh re-rendering. (a) Surface mesh re-rendering may result in enlarged face triangles. (b) Artifacts introduced in texture mapping due to enlarged triangles in the re-rendered surface mesh. Future implementation will mitigate these effects via interpolation of additional mesh vertices to break down the enlarged face triangles.

\section{Future Work}

The developed visualization tool allows real time display of changes to height, width, projection, and ptosis of breasts on the torso. While SPHARM parameters that regulate dimensions such as height, width and projection allow simulations in the overall size of the breast, subtle localized shape changes that can result from breast reconstruction procedures such as implant based or autologous reconstruction, are not adequately modeled. To accurately model localized changes in breast shape, additional SPHARM coefficients have to be identified and appropriate mathematical expressions for shape transformation have to be developed. In our future work, we will aim at identifying transformation functions for different breast surgeries that will enable simulation of surgery specific breast shape outcomes.

This work presents a framework for design and development of software for visualization and simulation of changes in breast size. We note that the simulation or display of surgical scars is not addressed here and will be critical in future work, especially for breast reconstruction surgery. Future work will focus on evaluating the visualization framework to assess surgeon and patient acceptance on usability and feasibility for clinical translation.

\section{Acknowledgement}

This work was supported by grants from the U.S. National Institutes of Health (R01CA143190 and R01CA203984). The data used in this study were collected in collaboration with the Department of Plastic Surgery at The University of Texas MD Anderson Cancer Center. The authors recognize the support and contributions of surgeon Mark T. Villa for patient images used in this study.

\section{References}

[1] D. A. Frederick et al., "The Barbie Mystique: Satisfaction with Breast Size and Shape across the Lifespan", International Journal of Sexual Health, Vol 20, 2008 - Issue 3. https://doi.org/10.1080/19317610802240170

[2] A. Donfrancesco et al., "Three-dimensional simulated images in breast augmentation surgery: an investigation of patients' satisfaction and the correlation between prediction and actual outcome.", Plast Reconstr Surg, 2013 Oct;132(4):810-22, https://doi.org/10.1097/PRS.0b013e3182a014cb.

[3] Tepper et al., "3D Analysis of Breast Augmentation Defines Operative Changes and Their Relationship to Implant Dimensions" in Annals of Plastic Surgery, May 2009. Vol 62 -Issue 5, p 570-575. https://doi.org/10.1097/SAP.0b013e31819faff9

[4] Liu C et al., "Measuring volumetric change after augmentation mammoplasty using a threedimensional scanning technique: An innovative method." Aesthetic Plast Surg. 2012;36:11341139. https://doi.org/10.1007/s00266-012-9944-8 
[5] Swanson E. A measurement system for evaluation of shape changes and proportions after cosmetic breast surgery. Plast Reconstr Surg. April 2012;129(4) :982-992. https://doi.org/10.1097/PRS.0b013e3182442290

[6] Oren $\mathrm{M}$ et al., "Three-Dimensional Imaging Provides Valuable Clinical Data to Aid in Unilateral Tissue Expander-Implant Breast Reconstruction", The breast journal, 2008. https://doi.org/10.1111/j.1524-4741.2008.00645.x

[7] L. Roose et al., "Simulation of Soft-Tissue Deformations for Breast Augmentation Planning." In: Harders M., Székely G. (eds) Biomedical Simulation. ISBMS 2006. Lecture Notes in Computer Science, Vol 4072. Springer, Berlin, Heidelberg. https://doi.org/10.1007/11790273 22

[8] P. Ciechomski et al., "Development and Implementation of a Web-Enabled 3D Consultation Tool for Breast Augmentation Surgery Based on 3D-Image Reconstruction of 2D Pictures", Journal of medical internet research, Feb 2012, 14(1): e21. https://doi.org/10.2196/imir.1903

[9] CANFIELD VECTRA 3D. https://www.canfieldsci.com/imaging-systems/vectra-xt-3d-imagingsystem/, accessed August 2019.

[10] ILLUSIO http://www.illusioimaging.com/surgeons.html , accessed August 2019.

[11] A. Cheong, "Computational modeling of breast shape using Spherical Harmonics," PhD Dissertation, Department of Electrical and Computer Engineering, University of Houston, 2018.

[12] L. Zhao et al., "Inferior breast-chest contour detection in 3-D images of the female torso," IEEE J. Transl. Eng. Heal. Med., 2016, Vol. 4, no, pp. 1-10. https://doi.org/10.1109/JTEHM.2016.2614518

[13] L. Shen et al., "Modelling three dimensional morphological structures using spherical harmonics", International Journal of Organic Evolution", April 20009, Vol. 63, Issue 4, pp 1003-1016. https://doi.org/10.1111/j.1558-5646.2008.00557.x

[14]C. Brechbühle et al., "Parametrization of closed surfaces for 3-d shape description," Comput. Vis. Image Underst., 1995, vol. 61, no. 2, pp. 154-170. https://doi.org/10.1006/cviu.1995.1013

[15]SPHARM-MAT http://www.iu.edu/ spharm/SPHARM-docs/C01 Introduction.htm/\#module-team, accessed August 2019.

[16] L. Shen and F. Makedon, "Spherical mapping for processing of 3D closed surfaces," Image Vis. Comput., 2006 vol. 24, no. 7, pp. 743-61. https://doi.org/10.1016/j.imavis.2006.01.011

[17]Visualization Toolkit, https://vtk.org/ accessed August 2019.

[18] Qt https://www.qt.io/ , accessed August 2019. 